\title{
Dissipative continuum model for self-organized pattern formation during ion-beam erosion
}

\author{
S. Facsko, ${ }^{*}$ T. Bobek, A. Stahl, and H. Kurz \\ Lehrstuhl und Institut für Halbleitertechnik, Rheinisch-Westfälische Technische Hochschule Aachen, \\ Sommerfeldstrasse 24, 52056 Aachen, Germany \\ T. Dekorsy \\ Institut für Ionenstrahlphysik und Materialforschung, Forschungszentrum Rossendorf, 01314 Dresden, Germany
}

\begin{abstract}
A continuum model, based on the damped Kuramoto-Sivashinsky equation, is shown to reproduce the morphology evolution during ion sputtering quite successfully. In a very narrow range of the damping parameter $\alpha$, the alignment of the structures into hexagonal domains is obtained under normal incidence of ions with striking resemblance to the experimentally observed dot patterns. The origin of this damping factor is discussed.
\end{abstract}

The detailed knowledge of the surface morphology induced by ion sputtering is of central importance for the formation of self-organized periodic patterns of nanostructures ${ }^{1}$ and for the exploration of fundamental limits in top down processes based on lithography and ion etching.

Recent attempts to explain the temporal evolution of dot patterns during ion sputtering by the undamped KuramotoSivashinsky (KS) equation failed in two decisive points., ${ }^{2,3}$ Firstly, they could not reproduce the hexagonal ordering of dots under normal incident ion sputtering (or off normal sputtering with simultaneous rotation of the sample) reported in the last years on III-V semiconductor and Si surfaces. ${ }^{1,4-6}$ Second, they did not predict the stabilization of the periodic patterns for long sputtering times. ${ }^{2,6}$ Since the ordering arises equally on different surface orientations and even on amorphous surfaces it can be concluded that the ordering into hexagonal domains does not depend on the initial crystal structure of the surface. Therefore, the ordering mechanism is inherent to the pattern formation process. ${ }^{7}$

In an extended numerical analysis of the long-time behavior of the two-dimensional (2D) damped KuramotoSivashinsky (DKS) equation Paniconi and Elder presented a stationary hexagonal ordered solution in the long-time limit that bears a striking resemblance with the dot patterns obtained by ion sputtering. ${ }^{8}$

The DKS equation applied for the surface morphology evolution during the erosion by ion beam sputtering under normal incidence is a partial differential equation for the surface height $h(x, y, t)$, with $x$ and $y$ lying in the surface plane:

$$
\frac{\partial h}{\partial t}=-v_{0}-\alpha h+\nu \nabla^{2} h-D_{\mathrm{eff}} \nabla^{4} h+\frac{\lambda}{2}(\nabla h)^{2}+\eta .
$$

Here, $v_{0}$ is the constant erosion velocity of the plane surface, $\nu$ is the "effective surface tension," which is caused by the erosion process and usually has a negative value leading to a primary (linear) surface instability., ${ }^{9,10}$ The diffusion coefficient $D_{\text {eff }}$, which is assumed isotropic, stands for the sum of all diffusion coefficients, i.e., the thermal diffusion ${ }^{9}$ and the erosion induced diffusion. ${ }^{5,11}$ Further on, the nonlinear term $\lambda / 2(\boldsymbol{\nabla} h)^{2}$ accounts for the tilt dependent sputtering yield and brings forth the saturation of the surface roughness in time. The time, where the surface roughness starts to saturate is referred to as the crossover time $t_{c}$. In the following, times smaller than $t_{c}$ are called early time (linear regime) and times larger than $t_{c}$ late time regime (nonlinear regime). The term $\eta$ accounts for the fluctuations introduced by the stochastic nature of the sputtering process ${ }^{12}$ and $-\alpha h$ introduces an additional dissipation suppressing spatiotemporal chaos under certain conditions. ${ }^{13}$

As Paniconi and Elder demonstrate, ${ }^{8}$ the numerical integration of Eq. (1) leads to three distinct solutions in the late time regime. On the variation of $\alpha$, the morphology change is reported as follows: for values of $\alpha$ only slightly smaller than a critical value $\alpha_{c},{ }^{17}$ highly regular patterns with large domains of perfect hexagonal ordering are obtained, separated by point and line defects. At smaller, intermediate values for $\alpha$ the authors find hexagonal patterns, where the dot structures breathe and oscillate in time, resulting in a morphology with less ordering. Finally, for small values of $\alpha$, spatiotemporal chaotic solutions are obtained, similar to the solutions of the undamped KS equation. ${ }^{14}$

Following the work in Ref. 8, the numerical integration of the DKS equation is performed on an equally spaced twodimensional mesh. Euler's method is applied for the time derivative and an isotropic form of the Laplace operator is applied, which includes eight nearest neighbors. For the numerical integration the following values for the coefficients were used: a mesh size of $400 \times 400$ points, a spatial step width $d x=0.5$, time steps $d t=0.01, \nu=-1, D_{\text {eff }}=1,2 \lambda$ $=0.01$, and for the fluctuations white noise with a maximum amplitude of $\eta=0.01$. In order to demonstrate the different dynamics of the morphology the coefficient $\alpha$ was varied from 0.15 to 0.26 , while keeping all other parameters constant. The critical value of $\alpha=\alpha_{c}$, where the dynamics changes from smoothing to roughening, evaluates for the above given coefficients to 0.25 . $^{8}$ The erosion velocity of the plane surface $v_{0}$ was eliminated in the simulations by the redefinition of $h=h-v_{0} t$.

In Fig. 1, the calculated pattern for $\alpha=0.24$ is compared with an experimental pattern, obtained on a $\mathrm{GaSb}(100)$ surface sputtered with $500 \mathrm{eV} \mathrm{Ar}^{+}$ions at an ion dose of 

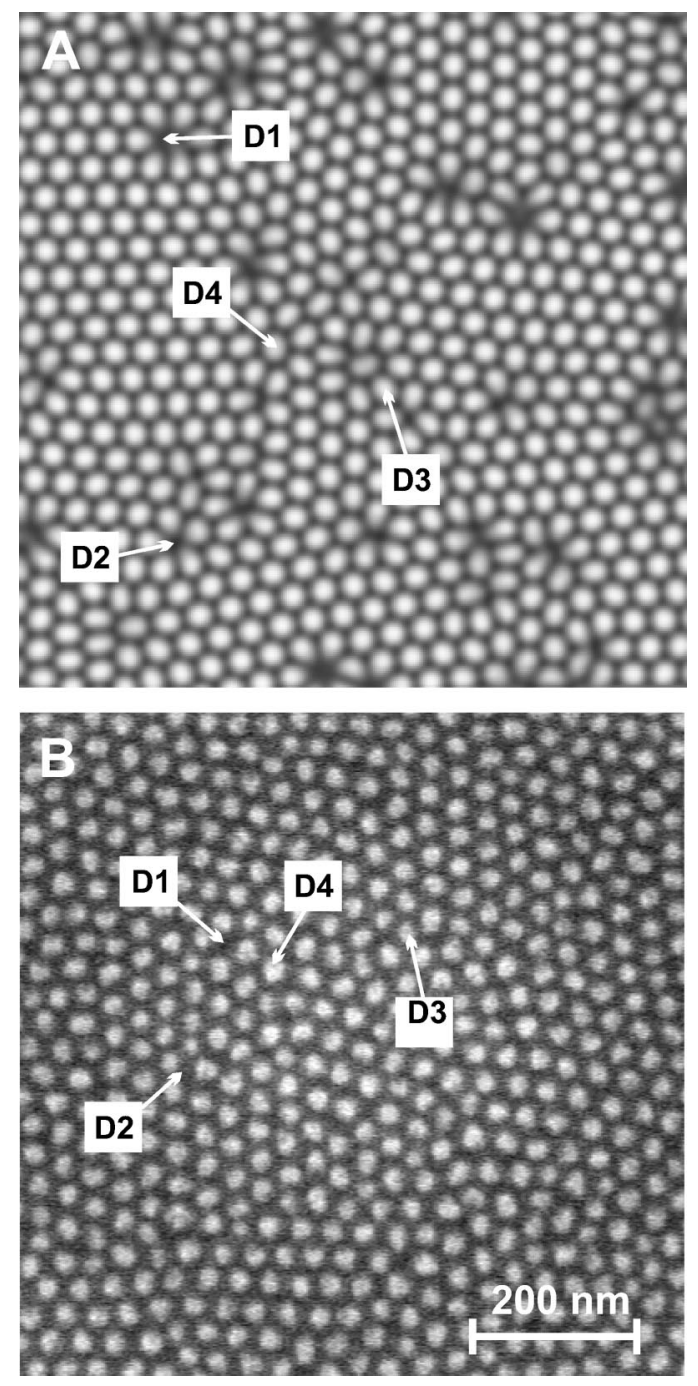

FIG. 1. Surface morphology after ion erosion: (a) Late time morphology calculated with the DKS equation with $\alpha=0.24$. (b) Scanning electron microscope image of a GaSb(100) surface after ion erosion with $500 \mathrm{eV} \mathrm{Ar}^{+}$ions with an ion dose of $2 \times 10^{18} \mathrm{~cm}^{-2}$. Similar defect types are labeled.

$2 \times 10^{18} \mathrm{~cm}^{2}$. In both cases the pattern consists of domains of hexagonally ordered dots with a domain extension of approximately eight lattice constants. The patterns are interrupted by "lattice defects" of the same types. The main defect types are a missing dot (vacancy) surrounded by six nearest neighbors (labeled D1), a missing dot with five neighbors (D2), a dot with five neighbors (D3), and a dot with seven neighbors (D4). There is an apparent difference in that the defects are more pronounced in the calculated pattern and more defects of type D1 are present. Nevertheless, from the temporal evolution of the calculated pattern it can be concluded, that this type of defect is not stable. For longer integration times, the amount of defects D1 decreases. Furthermore, for lower values of the parameter $\alpha$ the total amount of defects increases continuously. Finally, for $\alpha$ smaller than 0.2 , the hexagonal order disappears completely. Due to the lattice defects the hexagonal domains are oriented arbitrarily to each other and produce a pattern, which is iso-
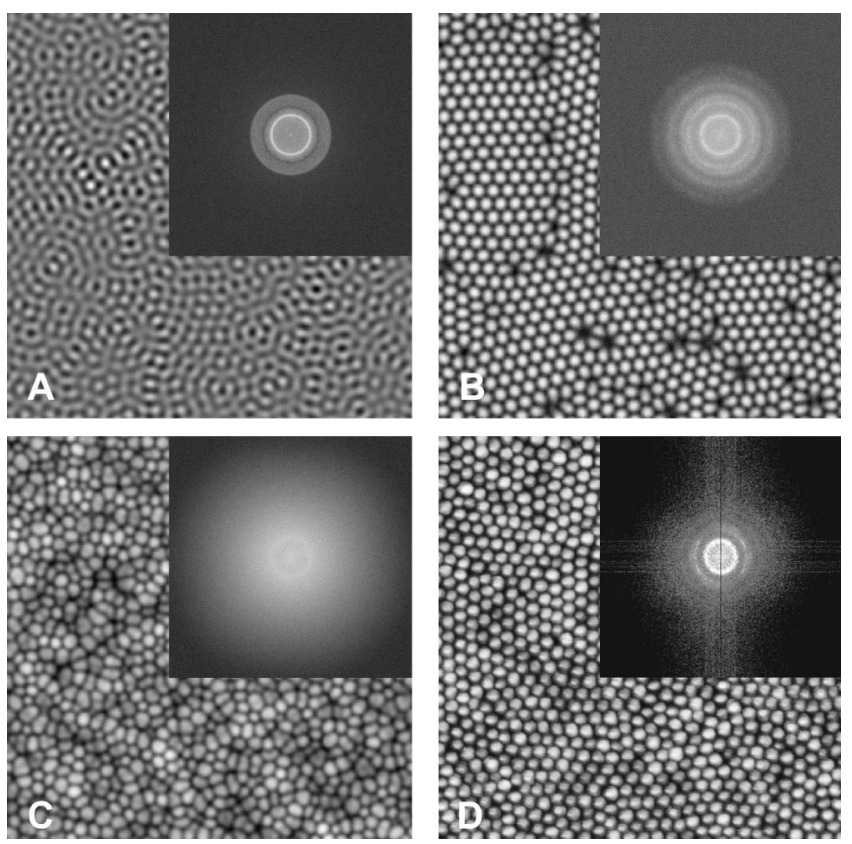

FIG. 2. Patterns calculated by the DKS equation with the corresponding 2D-PSD in the insets: (a) Pattern for the early time regime. (b) Stationary pattern with hexagonal ordering in the late time regime for $\alpha=0.24$. (c) Spatiotemporal chaotic pattern in the late time regime for $\alpha=0.15$. (d) AFM micrograph of a GaSb surface after sputtering with $500 \mathrm{eV} \mathrm{Ar}{ }^{+}$ions at $6 \times 10^{18} \mathrm{~cm}^{-2}$ ion dose, corresponding to the late time regime. The width of the micrograph is $125 \mu \mathrm{m}$.

tropic on a long-distance scale. The similarity between the experimental and simulated pattern with $\alpha=0.24$ emphasizes the importance of the stabilizing term $-\alpha h$, leading to the stationary solution in the form of hexagonally ordered dot patterns.

The origin of the hexagonal ordering is closely related to the damping term. In Fig. 2, three specific morphologies, which are solutions of the DKS equation and their 2D power spectral densities (PSD) are shown. These morphologies are compared to a micrograph of a GaSb surface, which was sputtered with $6 \times 10^{18} \mathrm{~cm}^{-2}, 500 \mathrm{eV} \mathrm{Ar}{ }^{+}$ions, determined by atomic force microscopy (AFM). In Fig. 2(a) the morphology calculated for the early time regime $\left(t<t_{c}\right)$ is shown for $\alpha=0.24$. In this linear regime no ordering is present, i.e., the pattern is fully isotropic and has only a characteristic periodicity. In this regime the pattern consists of chainlike structures, without clearly separated dots. In the crossover regime $\left(t \approx t_{c}\right)$, however, where the nonlinear term starts to be relevant, the dot structures develop and isolate, forming hexagonally ordered domains [Fig. 2(b)]. In this regime the individual dots appear to be "mobile" and can be rearranged, while keeping their shape. Therefore, the dots will be ordered such as mobile balls in a closed-packed arrangement. The different hexagonal domains appear at slightly different times, therefore being displaced to each other. The defects are created by rearrangements of the dots at the interfaces into metastable configurations. This hexagonally ordered pattern appears to be a stationary solution, in which only some of the defects of type D1 disappear in the 


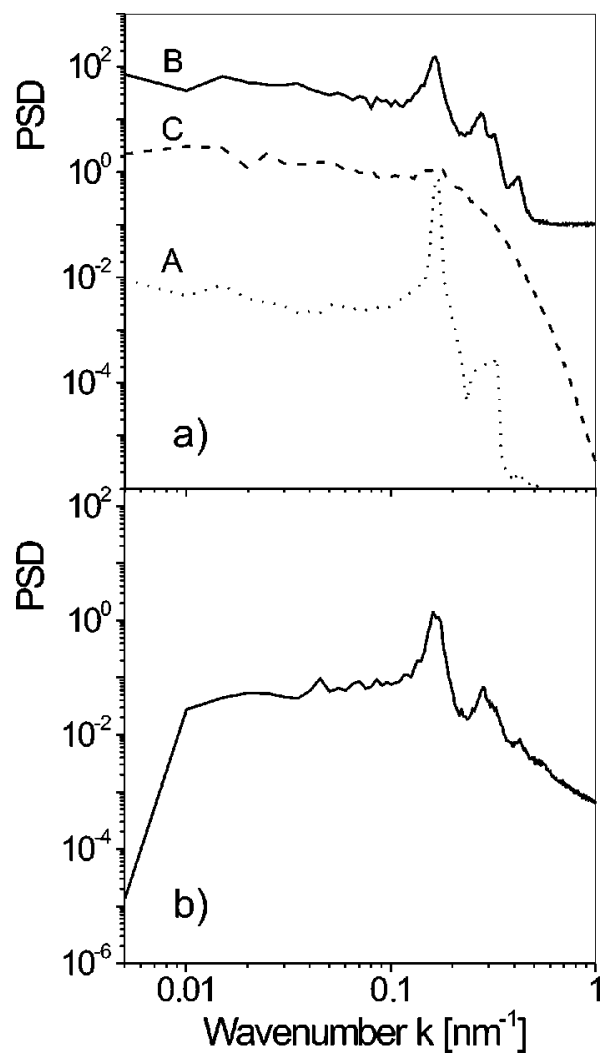

FIG. 3. Radial PSD's for the considered patterns of Fig. 2: (a) calculations: curve a, early time regime (dotted); curve b, stationary pattern for $\alpha=0.24$ in the late time regime (solid line); and curve c spatiotemporal chaotic solution for $\alpha=0.15$ in the late time regime (dashed). (b) PSD of the experimental pattern of a GaSb surface from Fig. 2(d).

long-time limit. For $\alpha$ smaller than 0.2 a spatiotemporal chaotic morphology appears, as shown in Fig. 2(c). The damping term $-\alpha h$ suppresses effectively secondary instabilities only for a narrow range of values of $\alpha \in(0.2,0.25)$.

The radial PSD's of the calculations, shown in Fig. 3(a), visualize the ordering phenomena and the distribution of the wave number in more detail. The curve for the early time regime (dotted line) already shows the predominant wave number, with a very small peak width. In the case of $\alpha$ $=0.24$, this peak stabilizes, the peak width increases slightly, and additionally, higher orders of the wave number ( $2 k$ and $3 k$ ) emerge in the late time regime (solid line). This indicates that the shape of the structures is nonsinusoidal, in agreement with the shape observed in the experiments on GaSb surfaces. ${ }^{1}$ For smaller values of the damping parameter $\alpha$ there is no predominant wave number visible any more in the late time regime (dashed line). The calculated PSD spectra are again in excellent agreement with the experimental data obtained by AFM measurements of GaSb surfaces ion eroded by $\mathrm{Ar}^{+}$ions of $500 \mathrm{eV}$ energy for $1200 \mathrm{~s}$ [see Fig. $3(b)]$.
In order to interpret the additional damping term $-\alpha h$ in the DKS equation, its microscopic origin must be addressed. In the following, we propose the most plausible microscopic mechanism and discuss its implications to the continuum equation. As the damping term depends on the surface position $h$ itself and not on derivatives of $h$, its origin cannot be traced to the deposition of the energy by the ion beam. ${ }^{10}$ Therefore, the damping term relies on a mechanism, that goes beyond the sputtering theory of Sigmund. ${ }^{15}$ An effect, which was ignored until now, but is known to play a significant role in the morphology of sputtered surfaces is the redeposition of the sputtered material. In the case of a heavily corrugated surface with high aspect ratio, a considerable amount of the sputtered particles hits the surface and is redeposited. This mechanism leads to a higher deposition rate in the depressions compared to the hillocks, therefore being a damping term in the case of ion erosion. There is also a net current of material from higher lying to lower lying regions, because of the forward peaking of the sputtered particle cloud in direction of the ion velocity vector, justifying the dependence on the height function $h$ itself. This effect has been observed recently in the evolution of the shape of V-grooves after high dose implantation. ${ }^{16}$ Because a generalized model including the particle redeposition would amount to a complex integro-differential equation, the damping term $-\alpha h$ has to be considered as a simple first approximation to include the redeposition by modulation of the erosion velocity $v_{0}$. While the original model leading to the KS-equation was based on the assumption of translational invariance in the $z$ direction, the damping term $-\alpha h$ obviously violates this symmetry requirement. But remembering the above interpretation of the damping as a simplified modelling of the redeposition process, one is led to a replacement of the simple term $-\alpha h$ by a term $-\alpha\left(h-\bar{h}_{A}\right)$, where $\bar{h}_{A}$ represents the height averaged over the area $A$. Under the assumption that $A$ is the whole grid area we have made sure that all results presented so far are not affected by this replacement. By this redefinition the DKS equation is transformed into a nonlocal continuum equation, indicating that the nonlocality is a necessary prerequisite for the ordering. The proposed mechanism should be verified experimentally and by molecular dynamic or Monte Carlo simulations.

In conclusion, the two-dimensional damped KuramotoSivashinsky equation reproduces quite successfully the formation of hexagonally ordered dot patterns on semiconductor surfaces during ion erosion at normal incidence, including the formation of the same types of lattice defects. These patterns occur particularly pronounced on GaSb and InP surfaces, and appear as a solution of the DKS equation in a narrow range of the damping parameter close to $\alpha=0.25$.

The authors acknowledge valuable discussions with $\mathrm{R}$. Cuerno, K.-H. Heinig, T. Müller, and O. Winkler. This work was performed with financial support from the Deutsche Forschungsgemeinschaft under Contract No. Ku 540/41. 
*Present address: Institut für Ionenstrahlphysik und Materialforschung, Forschungszentrum Rossendorf, P.O. Box 510119, 01314 Dresden, Germany. Electronic address: S.Facsko@ fz-rossendorf.de

${ }^{1}$ S. Facsko, T. Dekorsy, C. Koerdt, C. Trappe, H. Kurz, A. Vogt, and H. Hartnagel, Science 285, 1551 (1999).

${ }^{2}$ T. Bobek, S. Facsko, H. Kurz, T. Dekorsy, M. Xu, and C. Teichert, Phys. Rev. B 68, 085324 (2003).

${ }^{3}$ B. Kahng, H. Jeong, and A.-L. Barabási, Appl. Phys. Lett. 78, 805 (2001).

${ }^{4}$ F. Frost, A. Schindler, and F. Bigl, Phys. Rev. Lett. 85, 4116 (2000).

${ }^{5}$ S. Facsko, T. Dekorsy, and H. Kurz, Phys. Rev. B 63, 165329 (2001)

${ }^{6}$ R. Gago, L. Vazquez, R. Cuerno, M. Varela, C. Ballesteros, and J. Albella, Appl. Phys. Lett. 78, 3316 (2001).

${ }^{7}$ S. Facsko, T. Bobek, H. Kurz, T. Dekorsy, S. Kyrsta, and R. Cremer, Appl. Phys. Lett. 80, 130 (2002).
${ }^{8}$ M. Paniconi and K.R. Elder, Phys. Rev. E 56, 2713 (1997).

${ }^{9}$ R.M. Bradley and J.M.E. Harper, J. Vac. Sci. Technol. A 6, 2390 (1988).

${ }^{10}$ M. Makeev, R. Cuerno, and A.-L. Barabási, Nucl. Instrum. Methods Phys. Res. B 197, 185 (2002).

${ }^{11}$ M.A. Makeev and A.-L. Barabási, Appl. Phys. Lett. 71, 2800 (1997).

${ }^{12}$ R. Cuerno and A.-L. Barabási, Phys. Rev. Lett. 74, 4746 (1995).

${ }^{13}$ C. Misbah and A. Valance, Phys. Rev. E 49, 166 (1994).

${ }^{14}$ J.T. Drotar, Y.-P. Zhao, T.-M. Lu, and G.-C. Wang, Phys. Rev. E 59, 177 (1999).

${ }^{15}$ P. Sigmund, Phys. Rev. 184, 383 (1969).

${ }^{16}$ T. Müller, K.-H. Heinig, and B. Schmidt, Nucl. Instrum. Methods Phys. Res. B 178, 109 (2001).

${ }^{17}$ The critical value of $\alpha$ is defined as the value, where the primary instability occurs. When all coefficients are set to 1 in Eq. (1), $\alpha_{c}$ is $1 / 4$, as in Ref. 8 . 